\title{
Tensiones entre lo (i)legal y lo (i)legítimo en las prácticas de profesionales de la salud frente a mujeres en situación de aborto
}

\author{
Tensions between the (il)legal and the (il)legitimate \\ in professional health practices regarding women \\ who seek abortion
}

${ }^{1}$ Doctora en Ciencias Sociales. Coordinadora del Programa Género, Salud Reproductiva y Sexualidades, Instituto Psicología de la Salud, Facultad de Psicología, Universidad de la República, Montevideo, Uruguay. alopez@psico.edu.uy
Alejandra López Gómez ${ }^{1}$

RESUMEN La implementación de una estrategia sanitaria de atención pre y post aborto adoptada a partir del año 2004 en Uruguay, en un escenario legal restrictivo previo a su despenalización en 2012, abrió una ventana de oportunidad para vincular a las mujeres en situación de embarazo no deseado y aborto con los servicios de salud con el objetivo de disminuir su práctica insegura. En este contexto, este artículo busca indagar cuáles son y cómo operan las tensiones generadas por el cambio de un enfoque maternoinfantil hacia otro centrado en la salud y los derechos sexuales y reproductivos. A través de entrevistas semiestructuradas y grupos focales, se analizan las prácticas de los/as profesionales y sus esquemas de percepción y apreciaciones, en la atención a mujeres en situación de embarazo no deseado y aborto en los servicios del Sistema Nacional Integrado de Salud (SNIS) en Montevideo. Los resultados obtenidos brindan insumos para analizar algunas de las barreras y dificultades que se pueden observar actualmente en la implementación de la nueva ley.

PALABRAS CLAVES Salud Pública; Derechos Reproductivos; Servicios de Salud; Aborto Inducido; Aborto Ilegal; Uruguay.

\begin{abstract}
The implementation of a pre- and post-abortion health care strategy, adopted in 2004 in Uruguay within a restrictive legal context prior to the decriminalization of abortion in 2012, opened a window of opportunity to link women facing unwanted pregnancies and abortion to health services in order to prevent unsafe abortion practices. This article looks into the tensions generated by the change of focus from maternal-child health to health and sexual and reproductive rights, and how those tensions operate. Using semi-structured interviews and focus groups, the practices and perception and assessment frameworks of professionals in their care of women facing unwanted pregnancy and abortion in the National Integrated Health System in Montevideo are analyzed. The results offer insights into some of the barriers and difficulties that can currently be observed in the implementation of the new law.
\end{abstract}

KEY WORDS Public Health; Reproductive Rights; Health Services; Induced Abortion; Illegal Abortion; Uruguay. 


\section{INTRODUCCIÓN}

El aborto ilegal e inseguro es un asunto crítico en la gran mayoría de los países de América Latina y el Caribe, con consecuencias relevantes en el bienestar y la vida de las mujeres $^{(1),(2)}$. El avance en el reconocimiento político y social de los derechos sexuales y reproductivos como derechos humanos, que se registra en la arena internacional, regional y en escenarios nacionales, no ha estado ni está exento de disputas y controversias. El caso uruguayo ofrece importantes evidencias al respecto.

En el inicio del nuevo milenio, la sociedad uruguaya se involucró activamente en el debate sobre el derecho de las mujeres al aborto voluntario, en el marco más amplio de la discusión sobre la salud y los derechos sexuales y reproductivos. Por el impulso del movimiento feminista, el derecho al aborto seguro y legal se fue transformando en una plataforma ciudadana robusta en la que diversos actores articularon argumentos y acciones para incidir en el cambio del marco jurídico que penalizaba el aborto, así como en la respuesta institucional del sector salud frente a este problema ${ }^{(3),(4),(5)}$.

El sostenido debate social impactó en las políticas y los servicios de salud, y en las prácticas de los/as profesionales que en ellos se desempeñaban. La implementación de una estrategia sanitaria de atención pre y post aborto ${ }^{(6)}$, adoptada a partir del año 2004 por el Ministerio de Salud Pública mediante la Ordenanza $\mathrm{N}^{0} 369^{(7)}$, abrió una ventana de oportunidad para vincular a las mujeres en situación de embarazo no deseado y aborto con los servicios de salud, en un escenario legal restrictivo que tipificaba el aborto como delito. Esta normativa tuvo como cometido prevenir el aborto en condiciones de riesgo y reducir sus posibles daños, producto de la clandestinidad de la práctica. Constituyó la primera respuesta institucional del sector salud frente al problema del aborto inseguro mediante la intervención profesional antes y después del aborto, política que se fundamentó en el reconocimiento del derecho a la salud de las mujeres. La normativa ubicó el aborto inseguro como un problema de salud pública y su existencia interpeló el silencio histórico del sistema de salud ante esta realidad, el cual contó, a partir de ese momento, con un marco normativo para actuar, aunque fuera de manera limitada.

Podría decirse que se trató de una política de baja intensidad en la medida que: a) pocos servicios la implementaron y aquellos que sí lo hicieron la instrumentaron como prestación vertical no integrada a la atención en salud sexual y reproductiva; b) no fue monitoreada ni evaluada su implementación por parte de la autoridad sanitaria; c) no se desarrollaron estrategias de difusión hacia la población; y d) no incluyó criterios para la atención de abortos institucionales con base en las causales que definía la ley en ese entonces.

La salud y los derechos sexuales y reproductivos son un campo de disputa moral, ideológica, política, religiosa, simbólica y económica y, a la vez, de creciente legitimación y reconocimiento a nivel social. El aborto, como componente de la salud sexual y reproductiva, permite analizar una constelación de asuntos vinculados a la sexualidad, la (no) reproducción, el amor y la libre disposición y control del cuerpo de las mujeres -las principales involucradas-; cuestiones que remiten, en definitiva, a los conflictos entre lo individual y lo colectivo, lo público y lo privado, la universalidad y la particularidad, lo laico y lo confesional, la subordinación y la dominación, que se expresan en la atención de los servicios de salud y en el contrato entre profesionales y mujeres usuarias.

En el marco de una cultura patriarcal que se construye y reproduce en las prácticas profesionales, el modelomédicohegemónico ${ }^{(8),(9),(10)}$ ha sido base fundamental para el surgimiento y desarrollo del enfoque materno-infantil. Este modelo opera de manera eficiente no solo en la organización de los servicios y sus prestaciones tradicionales, sino en las prácticas profesionales, en sus posicionamientos subjetivos y en su formación técnico-profesional. El enfoque materno-infantil, sostenido 
en la ecuación mujer=madre, ha regido históricamente la definición de las políticas, los programas y la organización de los servicios de salud hacia las mujeres en Uruguay. La institucionalización de estas políticas, con una visión hegemónica del papel de las mujeres como cuidadoras de la salud familiar, en ausencia del varón y de su participación en los procesos reproductivos, trajo aparejadas dificultades para deconstruir y revisar los constructos de género en los que estas se basaban $^{(11)}$. La postulación de otro enfoque, el de la salud y los derechos sexuales y reproductivos ha interpelado, entre otros niveles, al de las prácticas profesionales de corte fundamentalmente biomédico.

Se pueden identificar tres planos interrelacionados en el campo de la atención sanitaria: 1) el de la definición de las políticas públicas, 2) el de la organización y gestión en los servicios de salud y 3) el de las prácticas profesionales $^{(12)}$. Estos planos deberían articularse de manera virtuosa para cumplir con el propósito de la atención integral cuando lo que se busca es garantizar derechos. Este modelo puede ser cuestionado por su carácter intrasistémico, si se considera que los servicios de salud son interpelados por las demandas ciudadanas y su exigibilidad de derechos.

Las prácticas profesionales pueden ser consideradas un analizador de las disputas morales e ideológicas que se registran a nivel social e institucional sobre el derecho de las mujeres a decidir sobre su (no) reproducción. Un analizador puede ser un elemento o situación de la realidad social (práctica, discurso, acontecimiento) que expresa las contradicciones del sistema permitiendo revelar la estructura de las instituciones, sus lógicas, sus normas, sus símbolos, su concepción del poder. El papel de los actores es clave en la medida que actúan en la situación desde la posición que ocupan en el sistema, al mismo tiempo que la situación actúa en ellos $^{(13)}$. Desde esta perspectiva, las prácticas de los/as profesionales frente a las mujeres y el aborto enuncian las contradicciones y complejidades que implica la incorporación de un enfoque de derechos y de género en el campo sanitario, construido desde una visión hegemónica biomédica y patriarcal.

Los modos en que los actores perciben, interpretan, aprecian y narran la realidad de la que forman parte, en nuestro caso, los esquemas de percepción y apreciación sobre sus prácticas profesionales, de acuerdo con Bourdieu, son producto del habitus, definido como representaciones sociales y prácticas incorporadas subjetivamente mediante procesos cognitivos y afectivos desde los cuales se produce y reproduce el sentido de la acción (profesional) y se recrea el campo (sanitario) y/o se lo transforma ${ }^{(14)}$. De allí la relevancia de analizar cómo los profesionales perciben sus prácticas profesionales, situadas institucional y sociohistóricamente.

La relación entre servicios de salud y mujeres en situación de aborto -relación mediada por el encuentro con los/as profesionales- es un locus privilegiado para evaluar las condiciones de posibilidad, dificultades y resistencias que encuentra la agenda de género y derechos en el campo sanitario. Dado que la estrategia sanitaria de prevención del aborto inseguro ha sido impulsada en otros países de la región donde existen condiciones legales restrictivas, el estudio aporta elementos para comprender algunos nudos críticos del papel de los/as profesionales en estos escenarios así como, eventualmente, para desarrollar acciones que permitan anticipar barreras y resistencias.

\section{El aborto, la salud y los derechos sexuales y reproductivos en Uruguay como objeto de la política}

La salud sexual y reproductiva constituye en la actualidad un campo interdisciplinario con un amplio repertorio de objetos de estudio, abordajes y diseños de investigación, metodologías y técnicas de intervención profesional y de acción política. En ella se entrecruzan aspectos políticos, científicos, sociales, éticos e ideológicos sobre el cuerpo, las sexualidades, la reproducción y la salud ${ }^{(15)}$.

La génesis de una política pública supone la asunción de un problema social. El proceso 
de delimitación y construcción del problema es parte del problema en sí mismo ${ }^{(16)}$. Entre 1996 y 2012, Uruguay avanzó de manera significativa al consagrar en su legislación un marco normativo que reconoce los derechos sexuales y reproductivos como derechos humanos y que ubica al Estado como su garante $^{[\mathrm{[a]}}$. Sin embargo, mantuvo el delito de aborto $^{[b]}$ en toda circunstancia hasta octubre de 2012 cuando tuvo lugar la aprobación de la Ley 18987 de Interrupción voluntaria del embarazo $^{(17)}$, la cual se puso en marcha en el Sistema Nacional Integrado de Salud a partir de enero de 2013. El cambio en la orientación de la política pública en salud sexual y reproductiva, desde mediados de los años noventa, se explica por el impacto de las conferencias de Naciones Unidas ${ }^{[c]}$ y por el trabajo de incidencia política de las organizaciones feministas ${ }^{(18)}$. Se definieron estrategias para la implementación de servicios de salud sexual y reproductiva en todo el país. En este proceso se han identificado obstáculos relacionados con barreras funcionales, organizativas, culturales y económicas que atentan contra el cambio de modelo de atención ${ }^{(19),(20),(21),(22) . ~}$ Estas barreras dan cuenta de los complejos procesos de transformación institucional y cultural que se requieren para reconocer a las mujeres como sujetos de derechos.

En este escenario, el aborto inseguro se instaló progresivamente en la agenda política como un problema de salud pública. La relación entre la definición de la política, su gestión en los servicios de salud y el nivel de las prácticas profesionales es un asunto que reviste enorme complejidad en sus múltiples conexiones y facetas. La consideración del aborto inseguro como problema de salud colonizó los debates parlamentarios que tuvieron lugar entre los años 2000 y 2012, siendo ésta la línea argumental de mayor peso -y el médico, la principal autoridad- a la hora de desplegar los fundamentos en uno u otro sentido. Si se analiza el conjunto de normativas sanitarias y actos administrativos aprobados a comienzos del nuevo milenio para regular la relación, respecto al aborto, entre médico/equipo de salud y usuarias, se observa una progresiva burocratización de los procedimientos y de las condiciones impuestas a las mujeres ${ }^{(23)}$. Al tiempo que estas medidas buscaron la apertura del sistema de salud a la realidad del aborto a través de la prevención de su práctica insegura, tuvieron como móvil restituir al poder médico su regulación, desde un discurso que combinaba los elementos tradicionales de la práctica médica y otro innovador, de reconocimiento de las mujeres como sujetos de derechos. Pero de derechos limitados a la intervención profesional, encargada de "velar" por una decisión de la mujer que fuera aceptable desde la perspectiva técnica, a través de dispositivos de asesoramiento.

El papel que jugó (y juega) la medicina en la construcción del problema del aborto no es novedad. Ello se inscribe en un proceso más amplio de medicalización de las sociedades occidentales que se inicia en el siglo XVIII y que tiene a la medicina como estrategia biopolítica de primer orden. Foucault ilustró magistralmente cómo el control social se impuso sobre los cuerpos concebidos como realidad biopolítica a través de la medicina como práctica social y, cómo el proceso de medicalización supuso la normalización de la medicina y de los médicos antes que de los pacientes ${ }^{(24)}$. La historia de los cuerpos no puede escribirse sin dejar de considerarse la ubicación de estos en el campo político. El autor señala que debemos abandonar la visión tradicional (e ingenua) que sostiene que el conocimiento se puede desarrollar por fuera de los requerimientos, las demandas y los intereses de poder ${ }^{(25)}$. La construcción sociohistórica de la medicina moderna y de la profesión médica ha sido ampliamente estudiada desde la sociología de la salud y la sociología de las profesiones. Al legado aportado por Foucault sobre el carácter social de la práctica médica se deben sumar, entre otros, los aportes de Canguilhem ${ }^{(26)}$, Boltanski ${ }^{(27)}$ e Illich ${ }^{(28)}$, quienes cuestionaron la visión tradicional de que la práctica médica se producía en relaciones e intercambios independientes de la vida social. Los estudios de Freidson ${ }^{(29),(30)}$, Zola $^{(31),(32)}$ y Turner ${ }^{(33)}$ son claves para analizar la relación entre medicina y sociedad, 
a través de cómo se configuran la profesión médica y las prácticas profesionales en salud. Por su parte, desde el pensamiento feminista se han realizado aportes ineludibles para un análisis crítico del papel de la medicina en el control y expropiación del cuerpo de las mujeres, en particular, de su autonomía reproductiva y capacidad de goce sexual(34),(35),(36).

Barrán $^{(37),(38)}$ estudió el papel de la medicina en la transformación de la sociedad uruguaya de comienzos del siglo XX mediante el análisis de cómo determinados asuntos sociales fueron construidos como problemas médicos mediante el otorgamiento de cientificidad a una estrategia higienista sobre el cuerpo y los intercambios humanos. La medicina fue una de las principales fuentes ideológicas en las que se sostuvo la nueva sensibilidad civilizada. El poder político y el poder médico se enlazaron estratégicamente para construir una cultura mediante el reconocimiento del monopolio médico sobre curar, medicar y certificar la normalidad. Esto implicó complejos procesos de producción de nuevas subjetividades subordinadas al saber-poder médico, con estrategias de clasificación de los/as pacientes en buenos o malos, puerilización y tutelaje ${ }^{(37),(38)}$.

A pesar de la creciente complejidad de la medicina contemporánea así como de la crisis que se puede observar en el contrato entre medicina y sociedad ${ }^{(39),(40)}$, la figura del médico continúa investida de un poder sobre los cuerpos y la vida que se puede observar, por ejemplo, en las múltiples maneras que adopta la violación de los derechos reproductivos en el ámbito de la salud ${ }^{(41),(42)}$. Como sostiene Tamayo, reconocer los derechos de las mujeres implica "establecer límites a la acción patrimonialista" o, mejor aún, denunciar y erradicar cualquier intento de dominación sobre las mujeres que busque despojarlas de la titularidad de derechos ${ }^{(43)}$.

\section{Cuerpo y reproducción: disputas en el campo de la salud}

La distinción entre sexo y género fue profusamente desarrollada desde el pensamiento feminista y ha sido y es objeto de intensos debates y revisiones. El uso extendido del concepto de género fuera de las fronteras del feminismo, su domesticación vía institucionalización en el campo de las políticas públicas, el riesgo de vaciamiento de su sentido político transformador y su naturalización pueden producir un efecto de debilitamiento de su eficacia política y analítica. Las frecuentes confusiones entre género y sexo; la homologación de género y mujeres; su asimilación al concepto de división sexual del trabajo de tradición marxista; el uso intercambiable con el concepto de patriarcado son algunos ejemplos de ello. Como ha dicho Scott, el "género se ha convertido en una forma cortés de referirse a cualquier cosa que tenga que ver con el sexo"(44 p.96).

El constructo naturaleza-cultura no es neutro para el género y las feministas buscaron cuestionar el determinismo biológico y su relación con la supuesta inferioridad de las mujeres y de lo femenino. Ortner ofreció una explicación sobre la dominación masculina, analizando el dualismo jerarquizante de la relación entre naturaleza y cultura para evidenciar las bases ontológicas de la lógica que presupone la inferioridad de las mujeres. Su planteo consistía básicamente en cuestionar que la base biológica que diferencia a mujeres y hombres fuera la causa de la inferioridad y la desvalorización de las mujeres y sostuvo que las mujeres habrían sido identificadas con la naturaleza, en contraste con los hombres asociados a la cultura ${ }^{(45),(46)}$.

En el centro de este constructo se encuentra el cuerpo de las mujeres, con un "privilegiado" aparato reproductor $y$, junto a él, la existencia de un "natural" instinto materno que determina sus conductas para hacerse cargo del producto de la procreación. La concepción naturalista opera de modo de obturar cualquier percepción sobre la variabilidad histórica y cultural de estos procesos, los cuales quedan sumergidos en la atemporalidad de los universales. Mediante complejas operaciones sociales y culturales, al ser la maternidad concebida como producto de la naturaleza, se erige como componente esencial de la feminidad ${ }^{(47)}$. 
El confinamiento de las mujeres al ámbito de lo privado tiene base en sus funciones reproductivas y de crianza, y la relación madre-hijo es presentada como un lazo natural que explicaría la omnipresencia materna en este ámbito. El ordenamiento social se basó en la construcción de un orden reproductivo, en un universo significante reproductor con base en la prescripción de normativas, sistemas de mandatos y prohibiciones y distribución de roles. Para cumplir con esta función de mediación, a través del maternaje es necesario producir unas subjetividades que la sostengan, es decir, una imagen de sí y del otro, unos modos de pensar y unos posicionamientos psíquicos con capacidad de apropiarse de ese "lugar" como si siempre hubiera estado ahí, despojándolo de toda percepción de construcción sociohistórica.

El concepto de género surgió enclaustrado en el binarismo naturaleza-cultura y buscó desmarcarse del determinismo biológico. Young afirma que la teoría de género surgió como un "gran relato", que buscó en sus inicios una explicación acabada y universal de la condición desigual de la mujer. Si bien ha sido y es cuestionable desde diversos ángulos, tuvo la virtud de producir nuevos argumentos que permitieron situar la problemática de la opresión de las mujeres en la agenda del debate político y teórico ${ }^{(48)}$. El género es una categoría inestable y cambiante, las críticas al concepto y sus refundaciones han surgido básicamente de las propias filas del feminismo. Estos cuestionamientos han estado centrados, fundamentalmente, en la crítica al binarismo sexo-género; a la supuesta existencia de dos géneros; a la construcción de la mujer y lo femenino como un todo cultural homogéneo y ahistórico; al rechazo a la concepción de la mujer-víctima y a la crítica a la heterosexualidad normativa obligatoria. El recorrido de construcción conceptual no ha sido lineal ni ha estado exento de tensiones ${ }^{(49)}$. Las transformaciones de la posmodernidad han producido nuevas prácticas y acuerdos sociales que confrontan el binarismo naturaleza-cultura y la dicotomía público-privado, generando nuevas significaciones que trastocan estos dominios. Sin embargo, las nuevas prácticas discursivas coexisten, no sin conflictos y tensiones, con las anteriores propias de las sociedades modernas, exigiendo ser situadas desde nuevos organizadores de sentido. Los nuevos modos de pensar la relación entre naturaleza y cultura, en cualquier caso, implicarán desterrar las operaciones de disyunción y reducción que han regido el pensamiento de tipo cartesiano. Es decir, el combate a la disociación ontológica entre naturaleza y cultura, base de la esencialización de las desigualdades sociales que se expresan a través del sexismo, la homofobia o el racismo; o a la disyunción entre mente y cuerpo; o a una concepción unívoca de la diferencia sexual. En términos del paradigma de la complejidad implica poner en juego operaciones de conjunción, distinción e implicación ${ }^{(50)}$.

La bibliografía feminista ha permitido alumbrar asuntos relevantes en el campo de la salud, cuestionando el androcentrismo de la ciencia médica y evidenciando la medicalización de las mujeres y sus padecimientos. Estos procesos no pueden considerarse sesgos o defectos de la medicina occidental, ya que lejos de "ser imperfecciones del sistema médico, se tratan de una cuestión estructural al propio sistema, que hay que recontextualizar y pensar en su totalidad"(36 p.35). En el campo de la medicina, se han hecho pasar por ciencia tres operaciones básicas del patriarcado que se han instrumentalizado, especialmente, sobre el cuerpo de las mujeres: la naturalización de la diferencia sexual como dato biológico inmutable e inferiorización de las mujeres, la fragmentación del cuerpo y la experiencia subjetiva, y la objetualización de los sujetos ${ }^{(35)}$. Tal como señala Giberti, es necesario producir un movimiento que posibilite la distinción de algunas implicaciones que han predominado en las teorías y prácticas en salud: a) desimplicar reproducción de maternidad; b) desimplicar reproducción de amor por la cría, y c) desimplicar maternidad de amor materno ${ }^{(51)}$. Estas tres fusiones han organizado las configuraciones de sentido de la acción profesional en el ámbito de la salud, y la decisión de abortar y el no deseo de hijo que expresan algunas mujeres trastocan estos dominios. 
Siguiendo el pensamiento de Bourdieu, la dominación masculina estructura el campo sanitario. Mediante complejos procesos la dominación se ancla en nuestro inconsciente, en las estructuras simbólicas y en las instituciones de la sociedad y se mantiene y reproduce mediante operaciones de coerción y consentimiento $^{(52)}$. Desde esta perspectiva es posible analizar cómo se crean, perpetúan y reproducen prácticas violentas en las instituciones de salud como parte estructural de un campo médico autoritario que son, la mayoría de las veces, "naturalmente" consideradas por los actores del campo como problemas de calidad de atención ${ }^{(53)}$. En este sentido, importa comprender cómo se organiza, estructura y opera el campo sanitario y el habitus profesional y de género y, cómo este enfrenta, acepta, resiste o recrea el discurso de los derechos reproductivos de las mujeres.

En este artículo se presentan algunos resultados obtenidos en un estudio sobre profesionales de la salud y aborto voluntario en Uruguay $^{[\mathrm{d}]}$ realizado en el escenario previo a la aprobación de la Ley 18987 de Interrupción voluntaria del embarazo que tuvo lugar en octubre de 2012, cuando el aborto era considerado un delito por la Ley 9763 de 1938.

\section{ABORDAJE METODOLÓGICO}

La pregunta principal que se buscó responder a través del estudio empírico fue: cuáles son y cómo operan en los servicios de salud las tensiones que genera el cambio desde un enfoque materno-infantil hacia otro centrado en la salud y los derechos sexuales y reproductivos que postulan las políticas de salud desarrolladas entre los años 2000 y 2012 en Uruguay. Para responderla, nos concentramos, a nivel micro, en el análisis de las prácticas de los/as profesionales, más específicamente en sus esquemas de percepción y apreciaciones, en la atención a mujeres en situación de embarazo no deseado y aborto en los servicios del Sistema Nacional Integrado de Salud (SNIS) en Montevideo. El estudio tuvo entre sus objetivos específicos analizar cómo se expresa la relación entre la definición de la política pública sobre aborto inseguro y su incorporación en la atención que brindan los/as profesionales en los servicios de salud.

Con base en estos objetivos, se definió una investigación de tipo descriptiva, exploratoria y analítica basada en una estrategia cualitativa. Para la recolección de la información, se utilizaron las técnicas de entrevista semiestructurada en profundidad y de grupo focal, en tanto vías para acceder a los esquemas de percepción y apreciaciones de los profesionales sobre sus prácticas, sus diferencias, controversias y similitudes en los modos de construir la relación con las mujeres que abortan y el sentido de sus prácticas de intervención técnico-profesional. Se diseñó una muestra intencional que incluyó a profesionales mujeres y hombres de distintas disciplinas vinculados a la atención en salud sexual y reproductiva, que se desempeñaban en los servicios del SNIS en Montevideo. Se realizaron 28 entrevistas y cinco grupos focales por tipo de profesión (enfermeros/as, médicos-ginecólogos/as, obstetras, parteras, psicólogos/as), a los cuales asistieron 39 profesionales. En total, participaron 67 profesionales, 41 mujeres y 26 hombres. De ellos, 32 eran médicos/as, 12 parteras, 12 psicólogos/ as y 11 enfermeras, con una media de edad de 42 años. El 60\% tenía más de 10 años de ejercicio profesional. La mitad de los/as profesionales se desempeñaba en ambos sectores (público y privado). El 60\% tenía hijos al momento de la entrevista. La mayoría se definió como ateo/agnóstico y quienes se identificaron con alguna religión lo hicieron mayoritariamente como católico no practicante. En términos de ideología política, la mayoría se ubicó en posiciones de centro y centro-izquierda.

Los participantes en el estudio lo hicieron de manera voluntaria habiendo recibido información sobre la investigación, tal como lo establece el Decreto CM/515/08 sobre Investigación con Seres Humanos. Se firmó un documento de consentimiento informado y se protegió la identidad de los/as participantes y la confidencialidad de la información durante todo el proceso de la investigación. 
El proyecto tuvo el aval del Comité de Ética en Investigación de la Facultad de Psicología de la Universidad de la República.

El tratamiento del material empírico siguió los procedimientos técnicos del análisis de contenido, desde un enfoque interpretativo de tipo hermenéutico-dialéctico, mediante el cual se buscó articular la comprensión de los procesos intersubjetivos, su contextualización y la visión crítica de los intereses en juego ${ }^{(54)}$. A partir de sucesivas lecturas del material y su codificación, se construyeron categorías operacionales o empíricas que fueron confrontadas con las categorías analíticas previamente definidas ${ }^{(55),(56)}$.

\section{RESULTADOS Y ANÁLISIS}

Este apartado se organiza en dos secciones. En la primera de ellas, se presentan algunos resultados sobre la relación entre lo (i)legal y lo (i)legítimo en la acción profesional frente al aborto. En articulación con lo anterior, la segunda sección analiza los supuestos de género que están en la base de los esquemas de percepción y apreciación de los profesionales sobre la legitimidad de la decisión de abortar de las mujeres. El análisis del material empírico a la luz de las consideraciones teóricas previamente enunciadas ofrece algunas pistas para comprender el complejo proceso que implica la transformación -en clave de género y derechos- de las prácticas profesionales e institucionales en el campo sociosanitario.

Siguiendo el pensamiento de Berger y Luckmann ${ }^{(57)}$, la función de la legitimación como productora de nuevos significados permite la incorporación del objeto como subjetivamente plausible. En este sentido, los autores señalan que:

...la legitimación produce nuevos significados que sirven para integrar los ya atribuidos a procesos institucionales dispares. La función de la legitimación consiste en lograr que las objetivaciones de "primer orden" ya institucionalizadas

\begin{abstract}
Ileguen a ser objetivamente disponibles y subjetivamente plausibles [...] Aquí, la cuestión de la plausibilidad se refiere al reconocimiento subjetivo de un sentido general "detrás" de los motivos situacionalmente predominantes, sólo parcialmente institucionalizados, tanto propios como ajenos. ${ }^{(57 \text { p.118-119) }}$
\end{abstract}

Los supuestos ontológicos, teóricos e ideológicos que orientan las percepciones y apreciaciones de los profesionales acerca de la (i)legitimidad de la intervención técnicoprofesional frente al aborto así como de la decisión de la mujer a interrumpir un embarazo son aspectos claves en el análisis de cómo se posicionan estos profesionales ante las mujeres que abortan, en términos del constructo de género desde el cual interpretan las decisiones (no) reproductivas de las mujeres y sus propias prácticas de intervención.

\section{Lo (i)legal y lo (i)legítimo: la acción profesional en el "borde"}

A partir del nuevo milenio, el amplio e intenso debate social sobre el derecho al aborto y las medidas programáticas adoptadas por la autoridad sanitaria para prevenir su práctica insegura tuvieron como efecto que el aborto inducido se transformara en un asunto para los servicios de salud, saliendo del secreto que lo había rodeado hasta ese entonces. A pesar de que el aborto era considerado un acto ilegal, comenzó a ser socialmente percibido como una decisión legítima o por lo menos aceptable. Su ilegitimidad había sido puesta en cuestión. Es interesante observar cómo esta tensión entre lo (i) legal y lo (i) legítimo era percibida y procesada -subjetivamente- por los/as profesionales. La normativa sanitaria pre y post aborto se sostenía en el reconocimiento explícito de este doble registro al legitimar la intervención técnica en un escenario jurídico que consideraba ilegal la práctica del aborto.

Lamas $^{(58)}$ sostiene que el aborto legal en el Distrito Federal de México, si bien ha 
permitido una conceptualización diferente sobre aborto y ha limitado las resistencias del personal de salud, per se no conlleva a la aceptación de la práctica, la cual estaría determinada por cómo se resuelve la confrontación entre el habitus médico y la legalidad. En el caso uruguayo, antes de la legalización de la interrupción voluntaria del embarazo, la tensión entre legitimidad e ilegalidad del aborto colocaba a los profesionales frente a ese fino límite de actuar en el borde, entre lo legal y lo ilegal. Mediante dispositivos de asesoramiento profesional, estos tenían el encargo institucional de informar y asesorar a las mujeres en la toma de la decisión, buscando "respetar" su decisión, luego de haber ofrecido alternativas frente al aborto. Si la decisión de la mujer era interrumpir su embarazo, los profesionales no podían realizar el aborto, sino solo informar sobre los métodos más seguros para ello. Tampoco podían extender recetas de la medicación: el misoprostol solo estaba autorizado en su uso intrahospitalario con fines obstétricos. La aprobación de la normativa generaba un escenario novedoso al favorecer el abordaje de estas situaciones pero implicaba restricciones en el accionar de los/as profesionales, lo cual les generaba incomodidad.

Para mi entender es hipócrita, porque te decimos todo lo que tenés que hacer pero no te damos nada. Arréglate como puedas de la puerta para afuera. Entonces creo que ahí estamos en un gran bache. Yo no puedo decirle a alguien "tenés que hacer esto, esto, esto", y "¿cómo lo hago?, "ah, yo no sé nada" y me lavo las manos. O no digo nada cómo hay que hacerlo o hago todo, pero no puedo quedarme en la mitad y largar a alguien por ahí, que es lo que se está haciendo ahora, largándolas por ahí [...] No hay mucho margen. La ley es muy clara, más allá que se esté de acuerdo o no, el aborto es ilegal. Sigue siendo delito... y el equipo de salud no tiene mucho marco para moverse. (Médico ginecólogo, 58 años)
Si bien la normativa sanitaria era valorada como una herramienta importante en la relación servicios de salud - usuarias, la percepción de los/as profesionales era que las autoridades y los mandos medios demostraban bajo compromiso con su aplicación. La mayoría de los servicios de salud no se habían organizado para brindar la prestación, no se disponía de lineamientos claros, no se habían instrumentado espacios de capacitación para el personal de salud de todos los servicios, ni se habían acordado las modalidades de intervención, lo cual habilitaba diferentes estrategias que cada profesional instrumentaba en función de su visión sobre el tema o, directamente, no se la aplicaba. La relación entre la definición de la política, su instrumentación en los servicios y su impacto en el cambio del modelo de atención materno-infantil era percibida como francamente deficitaria.

Si las instituciones tuvieran criterios más claros... Yo creo que eso haría permear mejor. Si fueran más claros los lineamientos, porque eso iría acompañado de capacitación y sensibilización del personal, no es simplemente "bajar el lineazo"... Porque muchas veces se ha hecho eso. Yo creo que eso es una de las cosas que faltan: un lineamiento y una directiva clara, desde lo que es la cabeza de la institución. Creo que facilitaría mucho las cosas, sobre todo porque eso tendría que ser informado al público quien luego va a usufructuar los servicios, entonces venís conociendo ya tus derechos. (Enfermera, 30 años)

Creo que el Ministerio dijo: "esto está muy bien, es una normativa, pero no hagan mucho ruido". Y me parece que una normativa de este tipo, para que tenga realmente impacto, requiere de la institución, porque parte del éxito es que las personas se empoderen de sus derechos, porque si no das la información necesaria. (Médica ginecóloga, 45 años) 
La aplicación de la normativa, al depender casi exclusivamente de la voluntad del profesional, abría espacio a un variado repertorio de modalidades de asesoramiento técnico. A partir del relato de los profesionales pudimos identificar tres modalidades, que hemos denominado: a) formal-instrumental: apegado al texto de la norma, con bajo involucramiento profesional, b) médico-normativo: interpretación restrictiva del texto de la norma, de tipo técnico-moral dependiente ${ }^{(39),(40)}$ y basada en una concepción de género que considera la reproducción y la maternidad como acto natural, y c) integral-implicado: interpretación amplia de la norma, en clave de respeto al derecho a la decisión de la mujer, con un alto involucramiento profesional. Estas modalidades no se configuraban en "estado puro" sino que coexistían de manera contradictoria en el relato sobre sus prácticas profesionales y daban cuenta de las controversias y disputas en la construcción del aborto (y de las mujeres que abortan) como objeto de intervención profesional.

\section{Hay profesionales que lo que hacen es con- denar y asesorar en cuanto a las opciones. Hay otros profesionales que no, sino que cargan de subjetivismo su recomendación o el análisis de la situación. Sigue depen- diendo mucho de la formación y de los valores, lo cual no debería ser así. (Médico ginecólogo, 44 años)}

A pesar de las limitaciones mencionadas, en un contexto en el que lo legal y lo legítimo disputan el escenario simbólico, los profesionales percibían ciertas transformaciones en los modos de relación entre servicios, profesionales y mujeres. Este proceso de cambio los implicó personalmente, "todos fuimos cambiando", en un proceso que no solo había involucrado a las mujeres, sino también a los/as profesionales -en particular a los más añosos- y a la interacción entre ambos.

Todos fuimos cambiando y, por ejemplo, si llega una mujer de 60 años y le pregunto si ha tenido abortos, demora en contestarme. Cuando son espontáneos los contesta enseguida... Sin embargo, si le pregunto a una mujer de 30 años me lo dice rápidamente y si le pregunto si fueron provocados, me lo dice rápidamente [...] La realidad social es totalmente distinta y todos vamos cambiando. Por lo tanto, creo que hoy una mujer que tiene veintitantos años, que tiene un embarazo no deseado, se acerca al equipo de salud a plantearlo, más allá de que termine o no en un aborto. Pero sí se acercan a plantearlo, mucho más de lo que era antes; antes, si no era deseado, pasaban lejos del equipo de salud porque solo querían llegar a quienes les practicaran el aborto; hoy se asesoran. (Médico ginecólogo, 58 años)

Los/as médicos/as más jóvenes y en proceso de formación vivían el nuevo escenario como un dato "natural", podían ser considerados/as "hijos/as de la normativa sanitaria": cuando se incorporaron a los servicios de salud, el aborto inseguro ya no era un problema ajeno a sus prácticas clínicas. A partir de esta experiencia, se posicionaban desde un lugar de "idealismo pragmático"(59) un punto de estrecha relación entre las prácticas y el contexto social, la estructura y la cultura institucional de la cual formaban parte.

\section{Es una cosa de todos los días entonces no Ilama la atención ni nada, salvo que apa- rezca muy avanzado el embarazo y que se lo haya provocado y ahí sí te llama la atención. Que ahí sí te puede dar a pensar algo pero si no, no; es normal, de todos los días que haya varios y a nadie le llama la atención. (Médico residente de Ginecología 1, 30 años, grupo focal)}

Los niveles de determinación de la práctica profesional son una conjunción de planos diferentes que remiten a lo social, lo político, lo ideológico, lo económico y lo subjetivo. La subjetividad implicada en el ejercicio profesional, los sistemas de valores y las concepciones sobre género, aborto, 
maternidad y derechos cobran enorme importancia en el proceso de implementación de las políticas públicas. La relevancia de este nivel estriba en el enlace potente que tiene la dimensión subjetiva con los esquemas de percepción y apreciación, en la medida en que reflejan los consensos, tensiones y controversias al interior de un colectivo en el que, si bien sus agentes ocupan distintas posiciones en el campo institucional, comparten lógicas, códigos, sistemas de reconocimiento, de legitimación y de sanción.

La tensión entre la ilegalidad del aborto y la legitimidad de la intervención profesional, antes de que este ocurra, es uno de los puntos que permiten comprender la baja implementación de esta política, desde las experiencias de los/as profesionales. Cabe preguntarse si el cuestionamiento de la legitimidad de la intervención -aun comprendiendo que se actuaba en "el borde" entre lo ilegal y lo legal- podría estar vinculado a una racionalidad que justifica las resistencias que los/as propios/as profesionales ponen en juego en el ejercicio de su rol, en particular cuando se trata de reconocer a las mujeres como sujetos con capacidad para tomar decisiones éticas sobre su vida reproductiva.

\section{El constructo "naturaleza-cultura" en el discurso profesional sobre el aborto}

El supuesto ontológico sobre el estatuto del aborto que se expresa en los discursos de los/as profesionales permitió identificar la relación entre el embarazo, considerado acto reproductivo-natural, y el aborto voluntario, en tanto decisión no reproductiva-antinatural. El embarazo y la maternidad quedan ubicados mediante complejas operaciones sociales y simbólicas como producto de la naturaleza, que definirá el destino ineludible y universal de las mujeres. A través de diferentes significantes, la relación naturaleza-cultura construye el discurso de los/as profesionales sobre el aborto. Estos significantes reproducen mandatos de género que justifican el tutelaje ${ }^{[e]}$ de las mujeres a través de la acción profesional, lo cual se observa, por ejemplo, en el asesoramiento pre-aborto de tipo médico-normativo. Al mismo tiempo, se registraron fisuras en los posicionamientos tradicionales y se identificaron nuevas producciones de sentido, las cuales se traducían, en ocasiones de manera precaria, en los modos de intervenir de los/as profesionales.

En los relatos, se advirtió la prevalencia de una narrativa principal que remite a la perspectiva ontológica sobre el "ser mujer" asociada a la maternidad como destino natural. En la concepción naturalista de las mujeres, de la que participan las creencias colectivas y los discursos científicos (entre ellos el discurso médico), se encuentran la noción de instinto y la ecuación mujer-madre-naturaleza, con efectos poderosos en la producción de los cuerpos y las subjetividades. Una visión que sostiene la irreductibilidad de la maternidad como núcleo central de la "identidad femenina".

Bueno, yo soy hincha de los bebés... El profesional la tiene que apoyar para que decida continuar con el embarazo, eso es lo natural, pero bueno, si decide interrumpirlo, ahí sí se le da la información sobre la medicación... no es cuestión de decirle "ah bueno, querés abortar, ta, tenés que tomar esta medicación y listo... no, no". (Médico ginecólogo, 63 años)

Como te dije, yo siempre estoy a favor de la vida. No te olvides que nosotros somos los que recibimos la vida. Yo participo, junto con los padres, de ese momento en el que las emociones están a flor de piel. El parto es un hecho lleno de emociones. (Médico ginecólogo, 52 años)

El "deseo natural" de tener un/a hijo/a puede encubrir motivaciones de las más diversas que se visten de maternidad, porque su prestigio y legitimidad social es aún incuestionable. Según Lamas, "la mujer tiene que dejar de interpretarse a sí misma como reproductora. O sea, cesar de aceptar, ahora sí, pasivamente, el acuerdo social acerca de su carácter esencialmente reproductor" ${ }^{\prime \prime 61}$ p.27). Esto mismo podría decirse de los/as profesionales. Aun 
en aquellos que reconocían el legítimo derecho de las mujeres a decidir sobre su vida reproductiva y su poder (de sí) para tomar la decisión, se pudo observar que la expectativa de cumplimiento del mandato social de la maternidad concebida como (hiper) responsabilidad, moralmente indeclinable y generizada tenía efectos poderosos a la hora de interpretar las prácticas sociales que desdicen este mandato.

\begin{abstract}
Entonces, claro, la vida te sensibiliza y te lleva a esto, a que la vida siempre, siempre es como una alegría, es como un placer, es como un... que te tira a más, siempre. Entonces, ante la intención de eliminar la vida, siempre a mí me causa un poco de... no sé cómo describirlo, me causa un cierto dolor, también. Pero, profesionalmente, trato de ser respetuoso de la decisión de la mujer, ¿no? (Médico ginecólogo, 58 años)
\end{abstract}

La vida, esa fuente "de placer, de alegría", está asociada a la capacidad procreadora del cuerpo de las mujeres. Cuando se enfrentan a situaciones de embarazo no deseado, algunos profesionales buscan estimular en la mujer su condición de ser "dadora de vida", ya que "para ello fuimos formados", dice un médico, o porque, como dijo una experimentada partera, "el parto es un acto lleno de emociones".

La vida en sí es un valor que tenemos que promoverlo. Yo como médico me formé para eso, salvaguardar la vida, el bienestar de las personas [...] Si una mujer se va a hacer un aborto tiene que ser consciente que dentro suyo hay una vida. Eso para mí es re importante, ¿no? (Médico ginecólogo, 45 años)

Las mujeres que deciden abortar asumen -la mayoría de las veces sin saberlo- un posicionamiento político no exento de costos sociales y emocionales. Teresa de Lauretis ${ }^{(62)}$ señala que la subjetividad se "engenera" mediante un mecanismo de compromiso subjetivo que las personas entablan con determinadas representaciones sociales. Estas subjetividades "engenerizadas" reproducen y también resisten, crean nuevas significaciones y prácticas enfrentando los discursos y dispositivos institucionales que, a su vez, las han producido. La decisión de interrumpir un embarazo puede ser interpretada como acto contrahegemónico, de resistencia, de autonomía y autorrepresentación, así como de transgresión de los mandatos de género: una transgresión a las "leyes de la naturaleza". Las mujeres que abortan son entonces consideradas por los/as profesionales como "desnaturalizadas", "irresponsables" o "valientes", dependiendo desde qué supuestos analizan esta decisión de las mujeres.
La maternidad como poder, como ben- dición, como... si abortás sos una hereje y creo que lo vas a seguir siendo, por más de que estemos en el siglo XXI... (Partera, 28 años)
Toda mujer sueña, en algún momento de su vida, con ser madre... (Médico gi- necólogo, 38 años)

El discurso médico, en tanto una de las tecnologías de género ${ }^{(62)}$ más poderosas de disciplinamiento, se enfrenta a estas "prácticas de sí" que despliegan las mujeres que deciden abortar. Su estrategia suele -bajo procedimientos y mecanismos cada vez más sofisticados y sutiles- resituar la domesticación del deseo de las mujeres y el tutelaje profesional a sus decisiones. El constructo embarazo $=$ vida $=$ felicidad $=$ celebración atraviesa el discurso de los/as profesionales. Como contrapartida, se encuentra la cadena de significantes aborto $=$ muerte $=$ dolor = trauma psíquico = censura. Estos significantes, ¿deben considerarse como un impensado de la práctica profesional? ¿Qué hacen (subjetivamente hablando) los/as profesionales con lo que les devuelve la realidad de sus "pacientes" y estas con lo que aquellos/as les proponen?

...vos que estuviste embarazada y que lo
amaste, es como que a veces te cuesta 
un poco. Yo igual asesoro pero diciendo "fa, es tan divino ser madre, por qué..." (Médica residente de Ginecología, 34 años, grupo focal)

Me da pena que esa mujer no pueda tener su hijo, por todo lo que representa, pero bueno uno tiene que respetar la decisión de cada paciente. Yo trato de ver, de conversar con ella qué posibilidades tiene de conservar ese embarazo, eso es lo primero que tiene que hacer. $Y$ uno empieza a escarbar y muchas de ellas, inconscientemente, querían quedar embarazadas... hay que tomarse un tiempito y conversar... (Médico ginecólogo, 62 años)

Mediante dispositivos de asesoramiento de tipo médico-normativo, se pedagogiza la decisión de la mujer, intentando disuadirla de la decisión de abortar. Así, el "deseo inconsciente" de embarazo se hace a la luz mediante la acción "técnica" que busca restituir el lugar de "la madre". El discurso médico como tecnología de género ha sido clave en la producción de significados cristalizados sobre las relaciones entre naturaleza y cultura, entre sexo y género, así como acerca del cuerpo y el determinismo biológico. Los/ as profesionales de la salud son portavoces de este discurso, no sin contradicciones, puesto que hoy disponen de un catálogo más amplio de significados de género y se vinculan con una diversidad de demandas de atención en salud, que provienen de subjetividades con mejores condiciones para cuestionar los sistemas dominantes sobre género y sexualidad.

\section{CONCLUSIONES}

Los resultados obtenidos contribuyen a comprender algunos de los nudos críticos implicados en la implementación de la atención pre y post aborto en Uruguay en un escenario legal restrictivo, previo a su legalización a fines del año 2012. La definición de la política pública de prevención del aborto inseguro evidenció algunas tensiones que no favorecían procesos de transformación en la organización de los servicios de salud, así como tampoco en los modos de intervención técnica, desde un enfoque de género y de derechos. El discurso de los/as profesionales, a través de un amplio repertorio de significantes, enunciaba la disputa simbólica sobre las mujeres, el cuerpo, las sexualidades y el aborto que se podían observar en el campo social, y que el debate público sobre los derechos sexuales y reproductivos había permitido visibilizar con mayor transparencia. Durante décadas, desde el campo médico, el aborto había sido construido como un problema del orden biológico, despojado de su dimensión social, política y ética. Se lo conceptualizaba como un acontecimiento involuntario, una externalidad generada por causas "naturales" que impedía concretar el instinto de maternidad de la mujer. Desde esta construcción del objeto profesional, el aborto no generaba incomodidades ni trastocaba las prácticas de los/as profesionales. Esta realidad comenzó a mutar cuando, a partir del nuevo milenio, el aborto pasó a ser considerado en tanto problema social y el campo de intervención profesional se trasladó del aborto, como objeto abstracto y a-social, al sujeto que decide abortar, es decir, las mujeres y sus circunstancias. Los constructos de género y las tecnologías derivadas de ellos producen los modos en que los/as profesionales perciben y aprecian a las mujeres que interrumpen voluntariamente el embarazo y determinan sus intervenciones.

Si género y sexualidad son considerados sistemas derivados de una compleja tecnología política, de aparatos biomédicos y dispositivos tecnosociales, estrategias de una biopolítica -al decir de Foucault- de la cual el poder médico es protagonista de primera línea, entonces, ¿es posible desde las lógicas establecidas en el campo médico dar lugar a prácticas con capacidad para enunciar otras relaciones de poder? ¿Qué condiciones se requieren para su transformación? Las respuestas a estas preguntas son fundamentales para hacer de la agenda de género y derechos 
una realidad en los servicios de salud y no solo un esfuerzo basado en el voluntarismo y compromiso de algunos/as profesionales. El campo médico, a pesar de sus resistencias, no es inmune a las transformaciones sociales de género, aunque predomine en su dinámica una cultura patriarcal que se expresa, por ejemplo, a través de los modos de construcción de sus objetos de intervención profesional.

La deconstrucción del modelo hegemónico materno-infantil exige desandar la ecuación simbólica mujer $=$ madre como un dato natural, para incluir las representaciones del deseo y del no deseo de hijo, ambas como legítimas producciones subjetivas y sociales.

El reconocimiento del derecho al aborto voluntario y su legalización trastoca las relaciones de dominación de género que se producen y reproducen en los distintos ámbitos sociales y que anidan en las subjetividades. Las dinámicas que se pueden observar en el espacio institucional de la salud son un ejemplo de ello. El nuevo escenario que inauguró la Ley 18987 de Interrupción voluntaria del embarazo en Uruguay, con sus limitaciones y controversias respecto al tutelaje a la decisión de las mujeres, ofrece un marco para analizar cómo impacta la legalidad de la práctica en la construcción social de la legitimidad de la decisión de la mujer y de la intervención técnico-profesional. Así se inaugura una agenda de investigación sobre la legalidad y la legitimidad del aborto voluntario que permitirá generar nuevas evidencias e interpretaciones sobre la compleja relación entre servicios de salud, profesionales y mujeres que deciden abortar.

\section{NOTAS FINALES}

a. En el año 2008 se aprobó la Ley 18426 de Defensa al Derecho a la Salud Sexual y Reproductiva, ley marco que reconoce los derechos sexuales y reproductivos como derechos humanos y que incluye, entre sus objetivos, la atención pre y post aborto.

b. La Ley 9763 de 1938 establecía el delito de aborto y la pena podía ser atenuada o eximida por el juez ante determinadas causales: violación, honor de la familia, angustia económica y riesgo de vida y salud grave de la mujer. La ley no fue implementada en los servicios de salud para la realización de abortos institucionales.

c. En particular la Conferencia Internacional sobre Población y Desarrollo (El Cairo, 1994) y la Cuarta Conferencia Mundial de la Mujer (Beijing, 1995),

d. El proyecto fue implementado inicialmente durante los años 2009 y 2010. Luego se continuó el trabajo de campo hasta avanzado el año 2011 en el marco de la Tesis Doctoral: "Profesionales de la salud y derechos reproductivos: transiciones y disputas en la atención a las mujeres en situación de aborto en Uruguay (2000-2012)", del Doctorado en Ciencias Sociales de la Facultad de Ciencias Sociales de la Universidad de Buenos Aires, defendida en mayo de 2015.

e. El derecho civil define "tutela" como la institución cuyo objeto es la guarda de las personas y bienes de quienes están bajo patria potestad o de aquellos que son incapaces de gobernarse por sí mismos. El tutelaje no es solo una forma jurídica o política. Es también un posicionamiento subjetivo que, como señala Amorós ${ }^{(60)}$, implica un escaso nivel de individuación. En el caso de las mujeres, un orden de prioridades puesto en el ser para otros, en el dar afectos y en el altruismo en detrimento del desarrollo personal. No serán pues intérpretes de sus propias necesidades, intereses, deseos, sino que requerirán que su voluntad sea interpretada por un otro. 


\section{AGRADECIMIENTOS}

El proyecto de investigación fue financiado por la Comisión Sectorial de Investigación Científica (proyectos I+D) de la Universidad de la República en Uruguay. Un especial agradecimiento a los y las profesionales que aceptaron participar de este estudio haciendo posible su concreción. La riqueza de sus relatos ha sido una fuente notable para comprender las vicisitudes y tensiones que atraviesan en sus prácticas profesionales cuando se trata de incorporar un enfoque de derechos humanos en el campo de la atención sanitaria a mujeres en situación de aborto.

\section{REFERENCIAS BIBLIOGRÁFICAS}

1. Department of Reproductive Health and Research, World Health Organization. Unsafe abortion: global and regional estimates of the incidence of unsafe abortion and associated mortality in 2008. 6th ed. Geneva: WHO; 2011.

2. Guttmacher Institute Facts on Induced Abortion Worldwide. Worldwide Incidence and Trends. New York: Guttmacher Institute; 2012.

3. Abracinskas L, López Gómez A. Mortalidad materna, salud y aborto en Uruguay. Montevideo: MYSU; 2004.

4. Abracinskas L, López Gómez A, (org). El aborto en debate: Dilemas y desafíos para el Uruguay democrático. Montevideo: MYSU; 2007.

5. Johnson N. El tratamiento de la despenalización del aborto en el ámbito político-parlamentario. En: Johnson N, López Gómez A, Sapriza G, Castro A, Arribeltz G, (coord). (Des)penalización del aborto en Uruguay: Abordaje interdisciplinario de una realidad compleja. Montevideo: CSIC, Universidad de la República; 2011.

6. Briozzo L. Aborto provocado - un problema humano: Perspectivas para su análisis - estrategias para su reducción. Revista Médica del Uruguay [Internet]. 2003 [citado 12 ago 2015];(19):188-200. Disponible en: http://goo.gl/WalSNk.

7. Ministerio de Salud Pública. Medidas de protección materna frente al aborto provocado en condiciones de riesgo, Ordenanza 369/04. Montevideo: MSP; 2004.

8. Menéndez EL. Hacia una práctica médica alternativa: hegemonía y autoatención (gestión) en salud. México DF: CIESAS; 1983.

9. Menéndez EL. Modelo Médico Hegemónico: transacciones y alternativas hacia una fundamentación teórica del modelo de auto atención en salud, en Arxiu D'etnografia de Catalunya: Revista D'antropologia Social. 1984;(3):83-119.

10. Martínez A. Antropología de la salud: Una aproximación genealógica. En: Prat J, Martínez A, (eds). Ensayos de Antropología cultural. Barcelona: Ariel Antropología; 1996. p. 369-381.
11. López Gómez A, Benia W, Contera M, Güida C. Del enfoque materno-infantil al enfoque de la salud reproductiva: Tensiones, obstáculos y perspectivas. Montevideo: Facultad de Psicología, Universidad de la República, Fundación Mexicana para la Salud; 2003.

12. Schraiber LB. Acción técnica y acción moral en los profesionales de la salud. En: Castro R, López Gómez A, (eds.). Poder médico y ciudadanía: El conflicto social de los profesionales de la salud con los derechos reproductivos en América Latina. Montevideo: Facultad de Psicología, Universidad de la República; CRIM-UNAM; 2010.

13. Lourau R. El análisis institucional. Buenos Aires: Amorrortu; 1994.

14. Bourdieu P. El sentido práctico. Buenos Aires: Siglo XXI Editores; 2007.

15. López Gómez A. Salud sexual y reproductiva en la agenda de investigación y formación en Psicología en Uruguay: Consideraciones conceptuales, nudos críticos y desafíos [Internet]. Montevideo: Conferencia Inaugural Académica, Facultad de Psicología, Universidad de la República; 2013 [citado 12 ago 2015]. Disponible en: http://goo.gl/Pi115i.

16. Parsons W. Políticas públicas: una introducción a la teoría y la práctica del análisis de políticas públicas. México: FLACSO; 2007.

17. López Gómez A. Aborto legal en Uruguay: el largo proceso para garantizar el acceso a la salud y los derechos reproductivos de las mujeres. En: Cavenaghi $S$, Cabella W, (org). Comportamiento reproductivo y fecundidad en América Latina: una agenda inconclusa. Río de Janeiro: ALAP; 2014.

18. López Gómez A, Abracinskas L. El debate social y político sobre la ley de defensa al derecho a la salud sexual y reproductiva en Uruguay. Montevideo: UNFPA; 2009 .

19. Mujer y Salud en Uruguay (MYSU), Observatorio Nacional en Género y Salud Sexual y Reproductiva. Informe 2010: Profesionales de la salud y salud sexual y reproductiva: Estudio sobre percepciones, valores y prácticas. Montevideo: MYSU; 2010.

20. Mujer y Salud en Uruguay (MYSU), Observatorio Nacional en Género y Salud Sexual y Reproductiva. Informe 2009. Montevideo: MYSU; 2009.

21. Mujer y Salud en Uruguay (MYSU), Observatorio Nacional en Género y Salud Sexual y Reproductiva. Informe 2008. Montevideo: MYSU; 2008.

22. López Gómez A, Carril E. Aborto voluntario y subjetividad en contextos de penalización: Efectos y significados en mujeres, varones y profesionales de la salud. Psicología, Conocimiento y Sociedad. 2010;1(2):36.

23. Sanseviero R. Barreras: Investigación y análisis sobre el acceso de las mujeres a decidir. Montevideo: Ruda, IPPF, AUPF; 2008. 
24. Foucault M. El nacimiento de la clínica: Una arqueología de la mirada médica. México: Siglo XXI Editores; 1983.

25. Foucault M. Microfísica do poder. 2a ed. Rio de Janeiro: Graal; 1984.

26. Canguilhem G. El conocimiento de la vida. Barcelona: Anagrama; 1976.

27. Boltanski L. Puericultura y moral de clase. Barcelona: Editorial Laia; 1974.

28. Illich I. The medicalization of life. Journal of Medical Ethics. 1975;1(2):73-77.

29. Freidson E. Profession of medicine: A study of the sociology of applied knowledge. New York: Dodd, Mead and Company; 1970.

30. Freidson E. Professional dominance: the social structure of medical care. New York: Atherton Press; 1970.

31. Zola I. La medicina como institución de control social. En: De la Cuesta Benjumea C, (comp). Salud y enfermedad: Lecturas básicas en sociología de la medicina. Medellín: Universidad de Antioquia; 1999. p. 23-46.

32. Zola I. Sendas que conducen al médico: Cómo una persona se convierte en paciente. En: De la Cuesta Benjumea C, (comp). Salud y enfermedad: Lecturas básicas en sociología de la medicina. Medellín: Universidad de Antioquia; 1999. p. 162-184.

33. Turner B. Profesiones, conocimiento y poder. En: De la Cuesta Benjumea C, (comp). Salud y enfermedad: Lecturas básicas en sociología de la medicina. Medellín: Universidad de Antioquia; 1999. p. 187-222.

34. Castro R, Bronfman M. Teoría feminista y sociología médica: bases para una discusión. Cadernos de Saúde Pública. 1993;9(3):375-394.

35. Valls-Llobet C. Mujeres, salud y poder. Madrid: Ediciones Cátedra; 2009.

36. Esteban ML. El género como categoría analítica: Revisiones y aplicaciones a la salud. Donostia-San Sebastián: Gakoa, Tercera Prensa; 1999.

37. Barrán JP. Historia de la sensibilidad en Uruguay: Tomo 2, La sociedad disciplinada. Montevideo: Ediciones de la Banda Oriental; 1993.

38. Barrán JP. Medicina y sociedad en el Uruguay del novecientos: El poder de curar. Montevideo: Ediciones de la Banda Oriental; 1992.

39. Schraiber LB. O médico e suas interações: a crise do vínculo de confiança. São Paulo: Hucitec; 2008.

40. Schraiber LB. Medicina tecnológica e prática profissional contemporânea: novos desafios, outros dilemas. Interface-Comunicação, Saúde, Educação. 1998;2(2):215-216.
41. Castro R. Génesis y práctica del habitus médico autoritario en México. Revista Mexicana de Sociología. 2014;76(2):167-197.

42. Chiarotti S, García Jurado M, Aucía A, Amihiardi S. Con todo al aire: Reporte de derechos humanos sobre atención en salud reproductiva en hospitales públicos. Rosario: INSGENAR, CLADEM; 2003.

43. Tamayo G. Bajo la piel: Derechos sexuales, derechos reproductivos. Lima: CMP Flora Tristán; 2001.

44. Scott J. Género: ¿todavía una categoría útil para el análisis? La Manzana de la Discordia. 2011;6(1):95-101.

45. Ortner S. ¿Es la mujer con respecto al hombre lo que la naturaleza con respecto a la cultura? En: Harris $\mathrm{O}$, Young K, (comp). Antropología y feminismo. Barcelona: Anagrama; 1979

46. Ortner S. Entonces, ies la mujer al hombre lo que la naturaleza a la cultura? Revista de Antropología Iberoamericana. 2006;1(1):12-21.

47. Fernández AM. La mujer de la ilusión: Pactos y contratos entre hombres y mujeres. Buenos Aires: Paidós; 1993.

48. Young IM. Vida política y diferencias de grupo: una crítica del ideal de ciudadanía universal. En: Castells C, (comp). Perspectiva feminista en teoría política. Barcelona: Paidós; 1996.

49. Flax J. Psicoanálisis y feminismo: Pensamientos fragmentarios. Madrid: Ediciones Cátedra, Universitat de Valencia; 1990.

50. Morin E. Introducción al pensamiento complejo Barcelona: Gedisa; 1992.

51. Giberti E. Parto sin temor: el poder que perdemos. En: Fernández AM, (comp). Las mujeres en la imaginación colectiva: Una historia de discriminación y resistencias. Buenos Aires: Paidós; 1992.

52. Bourdieu P. La dominación masculina. Barcelona: Anagrama; 2000.

53. Castro R. Indicios y probanzas de un habitus médico autoritario: el caso de las recomendaciones de las comisiones de derechos humanos en el campo de la salud reproductiva. En: Agoff MC, Casique L, Castro R, (coord). Visible en todas partes: Estudios sobre violencia contra las mujeres en múltiples ámbitos. México: Editorial Porrúa, CRIM-UNAM; 2013. p. 145-165.

54. Minayo MCS. La artesanía de la investigación cualitativa. Buenos Aires: Lugar Editorial; 2009.

55. Plummer K. Los documentos personales: Introducción a los problemas y la bibliografía del método humanista. Madrid: Siglo XXI Editores; 1989.

56. Miles M, Huberman M. Qualitative data analysis: A sourcebook of new methods. London: Sage Publications; 1984. 
57. Berger P, Luckmann T. La construcción social de la realidad. Buenos Aires: Editorial Amorrortu; 1998.

58. Lamas M. Entre el estigma y la ley: La interrupción legal del embarazo en el DF. Salud Pública de México. 2014;56(1):56-62.

59. Becker H, Geer B, Hughes E, Strauss A. Boy in White: Student Culture in Medical School. Chicago: University of Chicago; 1964.
60. Amorós C. Mujer: participación, cultura, política y Estado. Buenos Aires: Editorial de la Flor; 1990.

61. Lamas M. Política y reproducción: Aborto, en la frontera del derecho a decidir. México: Editorial Janés; 2001.

62. De Lauretis T. La tecnología del género. En: Diferencias. Etapas de un camino a través del feminismo. Madrid: Horas y Horas; 2000. p. 33-69.

\section{FORMA DE CITAR}

López Gómez A. Tensiones entre lo (i)legal y lo (i)legítimo en las prácticas de profesionales de la salud frente a mujeres en situación de aborto. Salud Colectiva. 2016;12(1):23-39.

Recibido: 10 de julio de 2015 | Versión final: 4 de octubre de 2015 | Aprobado: 1 de diciembre de 2015

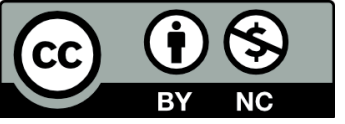

Este obra está bajo una licencia de Creative Commons Reconocimiento-NoComercial 4.0 Internacional. Reconocimiento - Permite copiar, distribuir y comunicar públicamente la obra. A cambio, se debe reconocer y citar al autor original. No Comercial - Esta obra no puede ser utilizada con finalidades comerciales, a menos que se obtenga el permiso.

http://dx.doi.org/10.18294/sc.2016.857 